\title{
A!
}

This is an electronic reprint of the original article.

This reprint may differ from the original in pagination and typographic detail.

Anis, Muhammad Moiz; Suarez, Luis; Leithon, Johann; Jayakody, Dushantha Nalin K.

\section{Multicast repair for broadcast flows to the handheld receivers}

Published in:

2018 IEEE Wireless Communications and Networking Conference, WCNC 2018

DOI:

10.1109/WCNC.2018.8377264

Published: 08/06/2018

Document Version

Peer reviewed version

Please cite the original version:

Anis, M. M., Suarez, L., Leithon, J., \& Jayakody, D. N. K. (2018). Multicast repair for broadcast flows to the handheld receivers. In 2018 IEEE Wireless Communications and Networking Conference, WCNC 2018 (Vol. 2018-April, pp. 1-6). (IEEE Wireless Communications and Networking Conference). IEEE.

https://doi.org/10.1109/WCNC.2018.8377264

This material is protected by copyright and other intellectual property rights, and duplication or sale of all or part of any of the repository collections is not permitted, except that material may be duplicated by you for your research use or educational purposes in electronic or print form. You must obtain permission for any other use. Electronic or print copies may not be offered, whether for sale or otherwise to anyone who is not an authorised user. 


\title{
Multicast Repair for Broadcast Flows to the Handheld Receivers
}

\author{
Muhammad Moiz Anis*, Luis Suárez ${ }^{\dagger}$, Johann Leithon ${ }^{\ddagger}$, Dushantha Nalin K. Jayakody ${ }^{\S}$ \\ ${ }^{*}$ School of Science and Engineering, Habib University, Karachi, Pakistan \\ ${ }^{\dagger}$ Huawei Technologies Co Ltd, Russian Research Center, Moscow, Russia \\ $\ddagger$ Department of Signal Processing and Acoustics, Aalto University, Espoo, Finland \\ $\S$ Institute of Cybernetics, National Research Polytechnic University, Tomsk, Russia \\ Email: moiz.anis@sse.habib.edu.pk, luis.suarez.rivera@huawei.com,johann.leithon@aalto.fi, nalin.jayakody@ieee.org
}

\begin{abstract}
Mobile TV receivers operate with a weaker link budget in the Digital Video Broadcast (DVB) network compared to the fixed rooftop mounted antenna receivers. Nevertheless, the cellular radio interface is an attractive alternative for broadcast services given its reliability. In light of the end-to-end service level convergence between the cellular and broadcast networks, we use Long Term Evolution (LTE) 's evolved Multimedia Broadcast Multicast Service (eMBMS) to multicast overhead parity packets among the multicast groups of mobile TV receivers (e.g. smartphones suffering from DVB packet erasures). The proposed multicast scheme recovers erased packets at each smartphone by Systematic RaptorQ Code (SRQC) decoding the directly received DVB transmission along with the overhead packets transmitted via eMBMS multicast. In this work we analyze the load generated in the Cellular Radio Access Network due to this erasure-recovery multicast scheme, and compare the gains of multicast over individual LTE unicast retransmissions of missing DVB packets.
\end{abstract}

Keywords-mobile TV; eMBMS; block codes; multicast repair; DVB-T2; LTE; real-time broadcast; multicast; linear combinations; erasure channel

\section{INTRODUCTION}

Mobile-TV broadcast has been an area of conflicting interests for the cellular and the broadcast networks' communities. The most recent terrestrial standard DVB-T2 includes some parameter's value-sets for handheld reception and also serves as a template for the latest DVB handheld transmission standard i.e. the DVB-Next Generation Handheld (DVB-NGH) [1-4]. DVB-NGH is a technically superior benchmark for contemporaries like Advanced Television Systems Committee (ATSC) 3.0. However, it has long been suffering from the commercial skepticism with no viable business plan. On the contrary, the $3^{r d}$ Generation Partnership Project (3GPP) eMBMS has just begun to receive some commercial acceptance.

In [4] authors advocate in favour of the end-to-end convergence of broadband and broadcast mechanisms yielding efficient mobile TV transmission. Later in [5] a cellular unicastbased Real-time Flow Repair (RFR) service was proposed which retransmitted lost DVB packets via LTE radio interface in a double coverage of DVB and LTE networks. In [6], a theoretical study in the posibilities of combining erasure coding techniques and retransmissions is presented. We got inspired from this work to provide an implementation point of view of the application of erasure coding for the specific scenario of DVB transmission to the LTE User Equipment (UE) e.g. smartphones. In the case of a large audience watching the same program, unicast based erasure-recovery may cause radio resource wastage for the retransmission of the same packets to multiple smartphones[5]. In this paper, we consider the loss of DVB packets in an erasure channel and analyze the repair transmission load in a cellular network, which uses multicast of common interest repair packets to a group of UEs. We consider an eMBMS Multicast bearer in the LTE Radio Access Network (RAN) transmitting linear combinations of DVB packets enabling a certain number of DVB erasure recovery at each member-smartphone of a multicast group. These linear combinations are the overhead parity packets generated by encoding the DVB transmission using SRQC. The systematically-generated overhead packets for each group of DVB packets per physical frame of the DVB transmission are transmitted via a multicast bearer in the LTE network. The number of overhead parity packets generated per group is a function of the code rate, which ultimately determines the number of recoverable erasures of the DVB packets for the multicast group receiving that physical frame. Each smartphone from a multicast group receives the primary DVB transmission from the DVB terrestrial network and processes each group of DVB packets with the corresponding parity packets through the repair multicast from the cellular network. With the multicast of common parity packets to a group of smartphones, the overall load is reduced on the LTE network with respect to the case of unicast repair, where every smartphone would get the lost DVB packets exclusively retransmitted via cellular network (See Figure 1).

In Section II our general proposal of an eMBMS-based erasure recovery scheme is presented. Section III discusses the use of SRQC for DVB erasure recovery. In Section IV, we explain our simulation model for a DVB Single Frequency Network (SFN), the concentric Multimedia Broadcast Single Frequency Networks (MBSFN) areas in the LTE networks and the erasure model for the DVB packets. In Section V an analysis of the simulation results obtained in our study is provided. Section VI concludes that the convergence between cellular and broadcast networks can yield some meaningful improvements in the performance if the multicast-erasure coding is used.

\section{EMBMS RECOVERING DVB PACKET ERASURES}

The eMBMS is a service used for the broadcast/multicast of video/multimedia content in the LTE network [7]. Its functioning depends on three architectural elements including 


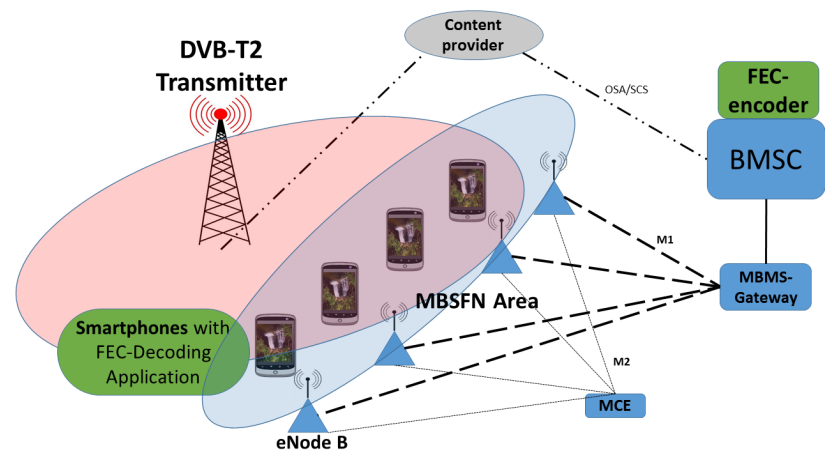

Fig. 1: FEC-based Erasure recovery in DVB transmission via eMBMS

a Broadcast Multimedia Service Center (BMSC), an MBMSGateway (MBMS-Gw) and an MBMS Coordination Entity (MCE). The BMSC is at the back of the LTE core network i.e. at the Evolved Packet Core (EPC). It serves all the multimedia broadcast/multimedia requests in the LTE network. Whereas, the MBMS-Gw and MCE reside inside the EPC. The MBMS-Gw is used to forward the packets, i.e. push data plane information in the eNode Bs participating in the broadcast/multicast. The MCE ensures a coordination among the concerned eNodeBs broadcasting the common content in their respective cells. An eNodeB is the building block of an eUTRAN $[8,9]$.

Primarily, EPC routes user data traffic between the eUTRAN and the IP Network, which is achieved by forming 'bearers' having a defined priority, delay tolerance, capacity and rate of packet loss for the data transmission via EPC [9]. Various services offer a certain set of preferred parametric values which determine the way an EPC shall assign network resources and schedule the given flow of data. Traditionally, there are some QoS templates used for the classification of a given bearer. In the case of eMBMS, the EPC negotiates among eNodeBs to form a multicast bearer. A multicast bearer has to be scheduled simultaneously for all the concerned eNodeBs having eMBMS clients in their cells.

In the DVB architecture we consider DVB-T2 transmitters to be the ultimate source of DVB transmission. DVB-T2 transmits data in Physical Layer Pipes (PLP), which allows to articulate the level of robustness for each broadcast data-flow. For example, a PLP aired for a mobile TV receiver, such as a smartphone, may be more protected by a lower Forward Error Correction (FEC) code rate. Each data flow is packetized in un-indexed BaseBand (BB)-frames prior to its FEC coding in the DVB-T2 data structure [10]. Each OFDM physical frame, namely DVB-T2 Frame carries several PLPs. A DVB receiver may separately decode each PLP's data-flow, as illustrated in Figure 2. For a given PLP, each T2-frame may yield a group of BB-frames decoded where some of them might be erased.

\section{A. Service and Procedures}

We propose to multicast/broadcast parity packets generated for the group of BB-frames of a particular PLP decoded from a given T2-frame of the DVB-T2 transmission. This multicast involves all eNodeBs to broadcast these parity packets, such that the number of parity packets decoded with the already

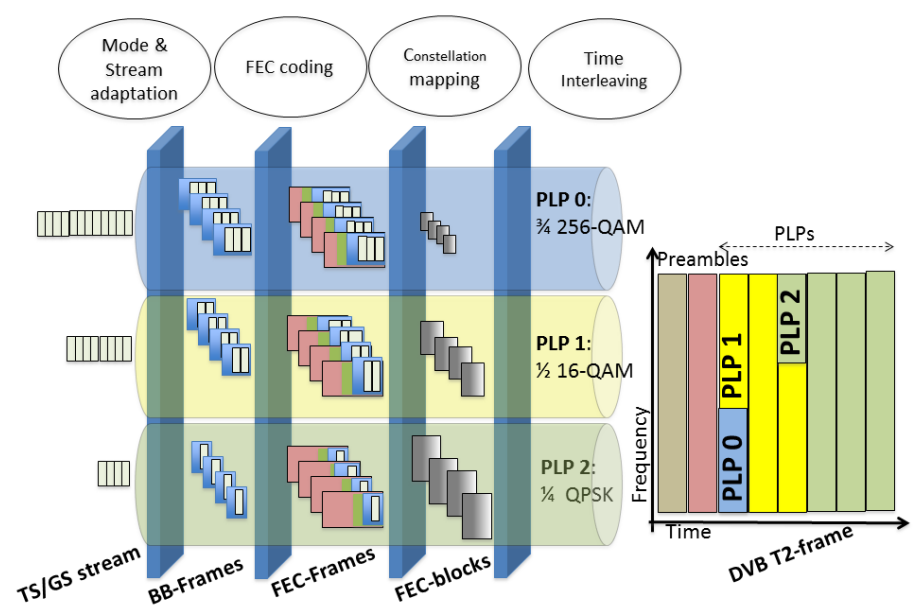

Fig. 2: DVB-T2 Data Structures

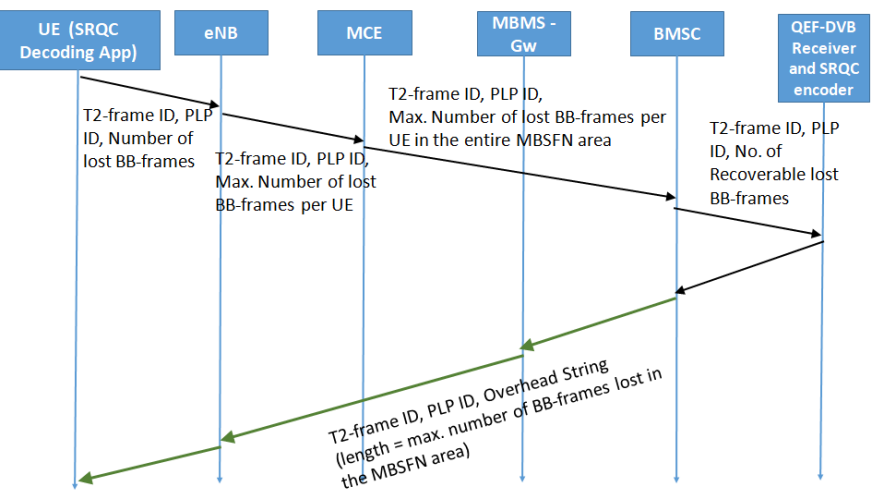

Fig. 3: BB-frame Erasure recovery mechanism via eMBMS

received $\mathrm{BB}$-frames from the principal DVB transmission is enough to recover a given number of erased DVB packets i.e. BB-frames. The proposed erasure-recovery operation consists of the following steps (also see Figure 3):

1) Each smartphone decodes the principal DVB transmission and experiences the erasures of some BBframes. The number of missing BB-frames is reported to the associated eNodeB by each smartphone receiving DVB transmission.

2) Following the reports from several Smartphones, the eNodeB reports to the BMSC about the maximum number of BB-frame erasures at any associated smartphone to the BMSC.

3) The BMSC algorithm and MCE schedule MBSFN area transmissions in the groups of eNodeBs, which have the same number of maximum erasures per smartphone. This multicast transmission comprises overhead parity packets assumed to be equal to the maximum number of erasures per smartphone in the MBSFN area. BMSC uses the Application LayerForward Error Correction (AL-FEC) i.e. SRQC encoder to generate the overhead packets.

4) The generated overhead packets are pushed to the 
member eNBs of the scheduled MBSFN area via the relevant MBMS-Gws. The eNodeBs belonging to the scheduled MBSFN area simultaneously air-out the parity packets strings for all User Equipments (UEs) i.e. smartphones.

5) Only subscribed smartphones are able to decipher the broadcast of the parity packets with the available key.

\section{MUlTiCAST OF THE OVERHEAD FOR THE SYSTEMATIC-RAPTORQ ENCODING}

Raptor Codes use a pre-coding step based on erasure correcting block codes. Then, the symbols generated with this pre-coding scheme are Luby Transform (LT)-coded. This step requires a degree distribution generator. The choice of an adequate pre-coding scheme may eliminate the need of a sophisticated degree distribution generator. Similarly, PreCodeOnly (PCO) Raptor codes can be realized by employing a complex precode. In [11], Shokrollahi proposed the Systematic Raptor codes, which can be used to generate an unbounded number of output symbols for any given input symbol. The Systematic Raptor encoding enables the regeneration of information/source symbols also among the encoded output symbols, which outnumber the inputs due to the overhead symbols. RaptorQ is the most recent Application Layer- Forward Error Correction (AL-FEC) code, which can encode several tens of thousand of source symbols, such that each symbol may be a long binary vector $[12,13]$. Traditionally the AL-FEC has been used for a reliable wireless multicast, by generating some output symbols greater in number than the source symbols.

In our work we consider a Systematic-RaptorQ Encoder (SRQE) and a roof-top mounted antenna DVB receiver embedded in the BMSC of the eMBMS architecture. The DVB decoder decodes all the DVB data flows and obtains the postdecoding BB-frames. The SRQE then encodes each group of BB-frames belonging to a specific PLP and a T2-frame index. The SRQE encoding systematically regenerates the BBframes along with some overhead frames. The overhead frames are specific to every individually scheduled MBSFN area ID, which is dynamically scheduled as a function of the collective channel conditions and corresponding maximum number erasures at any smartphone. In our solution we propose the multicast of overhead symbols only, for each of the multicast groups in the eUTRAN. Corresponding to every choice of the number of overhead symbols, a different decoding success probability can be realized [14]. Thus, different MBSFN areas may transmit a different number of overhead symbols such that a highly lossy multicast group requires the multicast of a higher number of overhead packets.

We use File Delivery over Unidirectional Transport (FLUTE) because of its multicast nature [15]. In order to achieve some synchronization with the DVB transmission sequence of the T2 frame index, we include some in-band signaling bytes along with the overhead symbols transmitted. The signaling bytes include T2-frame index and PLP-ID, in order to specify and synchronize the erasure-recovery operation for a given DVB broadcast data flow i.e. PLP and for a physical frame i.e. T2-frame. The inclusion of signaling along with the overhead parity packets helps the SRQ-FEC decoding application in using relevant overhead packets for determining the erased DVB-packets i.e. BB-frames, (see Figure 4).

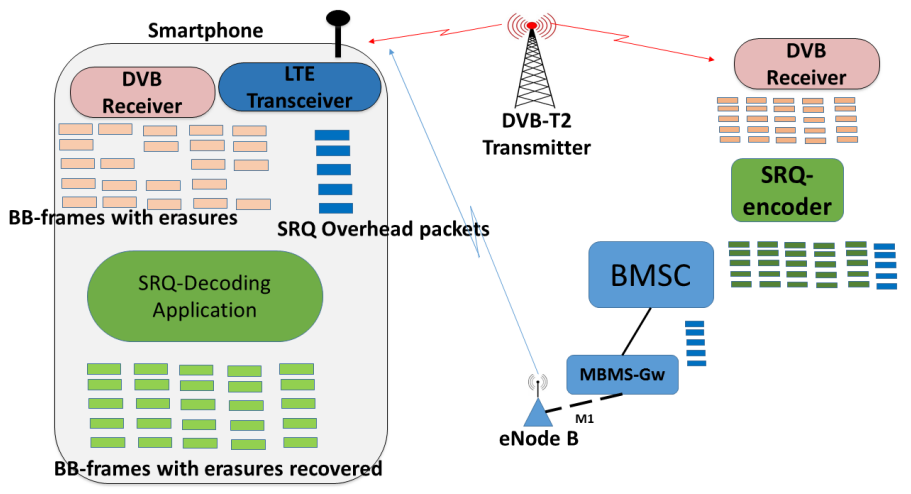

Fig. 4: Overhead packets generation and Erasure recovery via eMBMS.

\section{ERASUre Recovery Multicast Via Concentric MBSFN AREAS}

Single Frequency Networks (SFN) has been a classical method of improving radio link budgets for the DVB-T2 broadcast receivers. An SFN comprises several transmitters simultaneously transmitting the same signal at the same frequency, which a handheld receives with some diversity gain because of the resultant received signal being a recombination of the multiple receptions within a narrow time duration over the symbol duration. In an LTE eMBMS, broadcast/multicast also requires the temporary formation of SFNs known as Multimedia Broadcast Single Frequency Networks (MBSFN) areas. In order to form an MBSFN area in an eUTRAN, the MCE schedules a group of eNodeBs to disrupt their bidirectional signal exchanges and simultaneously transmit the same signal during the MBMS Broadcast subframe.

We consider a principal Digital TV transmission aired simultaneously from a central DVB-T2 transmitter and six other equidistant neighboring transmitters. The population is considered only in a large hexagonal cell around the central transmitter. The neighboring DVB-T2 transmitters contribute positively for the edge UEs but generate some self-interference for UEs inside the cell.The considered large hexagonal area bears a uniformly distributed population around the central transmitter of the DVB-T2 SFN. The considered population also experiences LTE coverage in the same region. The multicast bearers transmit SRQC parity packets, whose number depends on the maximum number of erased packets per device among all the smartphones in the MBSFN area. We consider concentric MBSFN areas which are comprised of all the eNodeBs located at the same distance from the central DVBT2 transmitter. In Figure 5 the small hexagons of common color represent the MBSFN ring formation for the equidistant eNodeBs, which are likely to remain equally loaded for the DVB transmission repair, because of the hexagonal symmetry around the central DVB-T2 transmitter.

\section{A. Load over eUTRAN}

We consider a variety of loading conditions in the eUTRAN by considering densely and lightly populated cases for a macrocell intersite distance. The results are obtained for concentric-MBSFN areas and the corresponding unicast NonSFN cases. The additional load over the eUTRAN is obtained 


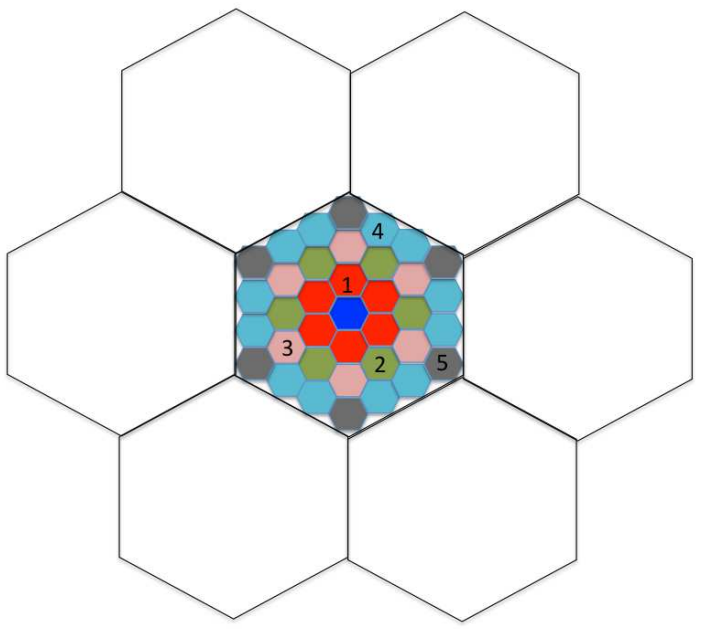

Fig. 5: DVB and LTE networks deployment [16]

through a DVB error performance model already presented in [16], which is analyzed in terms of the LTE resource allocation units.

\section{B. Erasure Model}

We consider the sigmoidal-curve-fit frame error model presented in [16] for the evaluation of the BB-Frame erasure rate. For this analysis we choose all parameters compliant with the T2-lite profile of DVB-T2 [17], [18]. A code rate of 0.5 for a short FEC frame has a BB-frame $b_{f r}=7032$ bits long $[10,16]$. We consider a source data-flow rate, $r_{\text {user }}=0.512$ Mbps [19], which is equivalent to $73 \mathrm{BB}$-frames per second.

\section{Load over the Multicast bearer}

For a given Signal to Interference Noise Ratio (SINR) of the DVB signal we evaluate the BER using the model approximated in [16]. The BB-frame Error Rate (FER) at each user multiplied by the data flow rate, $\frac{r_{u s e r}}{b_{f r}}$, gives the number of lost DVB packets i.e. BB-frames lost per user. In the case of unicast repair, an eNodeB retransmits the lost BB-frames summed for all the users in an LTE cell as the overall number of retransmissions of BB-frames per second per eNodeB, $L_{u n i}$, is given as,

$$
L_{\text {uni }}=\frac{r_{u s e r}}{b_{f r}} \sum_{i=1 \rightarrow n_{\text {percell }}} F E R_{i},
$$

where, $n_{\text {percell }}$ is the number of users per LTE cell and $F E R_{i}$ is the FER experienced by the $i^{t h}$ handheld receiver.

In this work, we consider that the LTE network performs SRQC encoding of the principal DVB transmission then multicast only the overhead parity packet string for the entire group of eNBs in an MBSFN area for the maximum number of lost BB-frames per smartphone, in a considered T2-frame of a given PLP. In this case, the load per eNodeB, $L_{m u l}$, in contrast to (1) can be given as,

$$
L_{m u l}=S R Q C\left[\max _{i=1 \rightarrow n_{\text {percell }}}\left(\frac{r_{u s e r}}{b_{f r}} F E R_{i}\right)\right],
$$

TABLE I: DVB-T2 Parameters [16]

\begin{tabular}{|l|l|l|l|}
\hline \multicolumn{2}{|l|}{ Parameter } & Value & Unit \\
\hline Transmission Power & $P_{t}$ & 68 & $\mathrm{dBm}$ \\
\hline Inter-Site Distance & $D$ & 52 & $\mathrm{~km}$ \\
\hline $\begin{array}{l}\text { DVB Transmitter } \\
\text { height }\end{array}$ & $h_{D V B}$ & 150 & $\mathrm{~m}$ \\
\hline Smartphone height & $h_{r}$ & 1.5 & $\mathrm{~m}$ \\
\hline Antenna Gain & $G_{\text {iso }}$ & -5 & $\mathrm{dBi}$ \\
\hline Carrier Frequency & $f_{c}$ & 650 & $\mathrm{MHz}$ \\
\hline PLP data rate & $r_{\text {user }}$ & 0.512 & $\mathrm{Mbps}$ \\
\hline
\end{tabular}

where $S R Q C[x]$ gives the number of bits of the SRQC overhead parity packets for a number $x$ of BB-frames getting erased. Notice that in this case $x$ corresponds to the maximum number of lost BB-frames per smartphone found among the whole group of users in the MBSFN.

\section{Number of the Pairs of Resource Blocks}

In [20] authors define the spectral efficiency using Shannon formula for the considered LTE network parameters (see Table III). the spectral efficiency, $S_{i}$, is given as,

$$
S_{i}=0.56 \log _{2}\left(1+\gamma_{i} / 2\right)
$$

where $\gamma_{i}$ is the SINR LTE signal received by the $i^{t h}$ smartphone. The available capacity at an eNodeB is measured in terms of the maximum number of Pairs of Resource Blocks per Transmission Time Interval (PRBs per TTI). A PRB has a bandwidth of $B_{P R B}=0.180 \mathrm{MHz}$, which makes it $50 \mathrm{PRBs}$ per TTI for $10 \mathrm{MHz}$ [8].

From (2) the bits per second to be commonly transmitted for all the smartphones receiving a multicast of parity packets from eNodeB is $S R Q C\left[\max _{i=1 \rightarrow n_{\text {percell }}}\left(r_{\text {user }} F E R_{i}\right)\right]$. Thus, the number of PRBs occupied per TTI by the proposed erasure-recovery mechanism at each eNodeB can be given as,

$$
P R B_{e N B}=\frac{S R Q C\left[\max _{i=1 \rightarrow n_{\text {percell }}}\left(r_{\text {user }} F E R_{i}\right)\right]}{\min _{j=1 \rightarrow n_{\text {percell }}} S_{j} \cdot B_{P R B}} .
$$

The number of bits carried by each PRB in a TTI depends on the spectral efficiency available. Notice that the worst case spectral efficiency is considered, which gives the maximum number of PRBs (i.e. Bandwidth) required for the multicast repair in an MBSFN area.

\section{PARAMETERiC VAlues AND Simulation RESUlts}

We consider a double coverage i.e. DVB-LTE, for a moderately and a densely populated scenarios, where $2 \%$ of users subscribe for multicast-erasure recovery via eMBMS-LTE. Considering that the subscribers base is equally divided among four network operators, the effective population densities are 2 and 12.5 subscribers per sq. $\mathrm{km}$. See Tables I, II and III.

Two or more eNodeBs associated to the group of subscribers which are equally far from the DVB transmitter will have similar PRBs per TTI loading. Thanks to the hexagonal symmetry in the DVB and LTE simulation networks, equally 


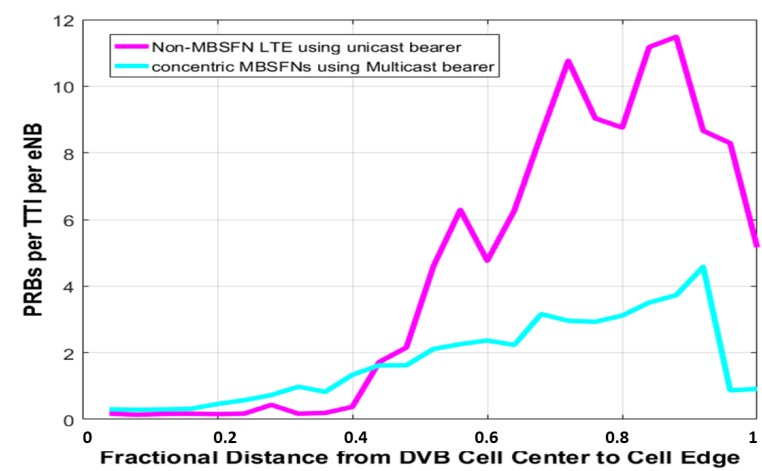

(a)

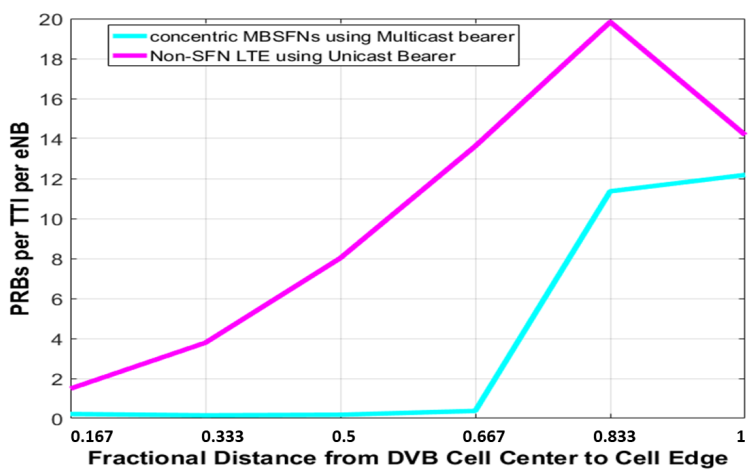

(b)

Fig. 6: MBSFN Resource efficiency a) for a moderately populated scenario b) for a densely populated scenario

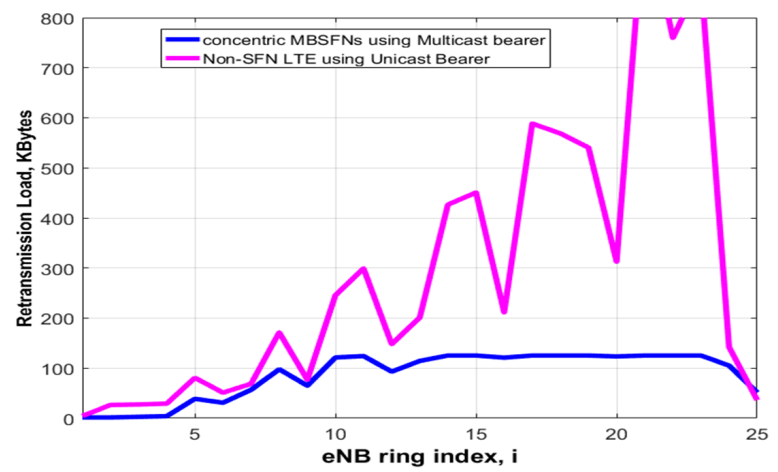

(a)

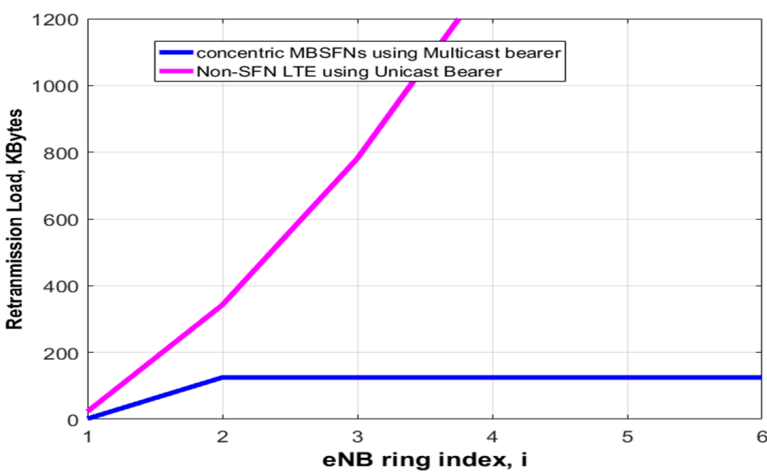

(b)

Fig. 7: Total retransmission $\mathrm{KBs}$ in an MBSFN ring for a) $\mathrm{ISD}=3.46 \mathrm{~km} \mathrm{~b}$ ) $\mathrm{ISD}=8.66 \mathrm{~km}$

TABLE II: Subscribers' Population Densities

\begin{tabular}{|l|l|l|l|}
\hline \multicolumn{2}{|l|}{ Parameter } & Value & Unit \\
\hline Population densities & $\rho$ & $\begin{array}{l}400, \\
2500\end{array}$ & $\begin{array}{l}\text { per sq. } \\
\mathrm{km}\end{array}$ \\
\hline Subcribers Percentage & $p$ & $2 \%$ & - \\
\hline Number of Operators & $n_{N O}$ & 4 & - \\
\hline $\begin{array}{l}\text { Subcribers Population } \\
\text { densities }\end{array}$ & $\rho_{\text {eff }}$ & $2,12.5$ & $\begin{array}{l}\text { per sq. } \\
\mathrm{km}\end{array}$ \\
\hline
\end{tabular}

loaded eNodeBs are likely to form concentric rings of equisdistant eNodeBs around the DVB-T2 transmitter. Therefore in our simulations we consider concentric MBSFN areas around in the DVB cell (see Figure 5). In our simulations we consider a single DVB cell, where eNodeBs surround the central DVB transmitter as MBSFN rings such that each ring temporarily transmits a common string of overhead parity packets in all of the LTE cells, for a commonly aligned and scheduled time slot in the LTE frames. Otherwise the eNodeBs keep transceiving the routine exchanges. We identify each MBSFN with an index number for the ring. The index equal to 1 represents the closest ring comprising the first six eNodeBs around the DVB transmitter.
TABLE III: Parametric Values for LTE Network [8]

\begin{tabular}{|l|l|l|l|}
\hline \multicolumn{2}{|l|}{ Parameter } & Value & Unit \\
\hline Transmission Power & $P_{t}$ & 49 & $\mathrm{dBm}$ \\
\hline eNB antenna gain & $A$ & 17 & $\mathrm{~dB}$ \\
\hline Inter-Site Distance & $I S D$ & 1.73 & $\mathrm{~km}$ \\
\hline Bandwidth & $B$ & 10 & $\mathrm{MHz}$ \\
\hline Carrier Frequency & $f_{c}$ & 2100 & $\mathrm{MHz}$ \\
\hline Receiver antenna Gain & $G_{i s o}$ & 0 & $\mathrm{dBi}$ \\
\hline Smartphone height & $h_{r}$ & 1.5 & $\mathrm{~m}$ \\
\hline eNB antenna height & $h_{e N B}$ & 30 & $\mathrm{~m}$ \\
\hline
\end{tabular}

In Figures 6.a and 6.b we present the required LTE capacity for a DVB retransmission with respect to the eNodeB ring index. We consider an Inter-site Distance (ISD) for the LTE network to be equal to $1.73 \mathrm{~km}$. For the case of a moderately populated scenario in Figure 6.a, the required LTE network capacity is 12 PRBs per TTI i.e. $24 \%$ of the total capacity when the Non-SFN unicast bearer is employed, whereas this number is reduced to 4 PRBs for multicast repair. For a densely populated scenario i.e. Figure 6.b, the required LTE capacity for the MBSFN case is even less than $2 \%$ of the total capacity 
at a distance just inside the cell edge (i.e. eNodeB ring index 4) of the DVB cell. As mentioned previously, we consider the weakest Spectral Efficiency for a smartphone in the entire MB$\mathrm{SFN}$ area. In spite of an inflated radio resource allocation for all practical reasons, the multicast repair operation is multiplefolds lighter compared to the unicast alternative. Notice also that multicast repair load is smooth as a function of distance contrary to the unicast situation.

In Figures 7.a and 7.b we evaluate the amount of data (in KiloBytes) to be transmitted for ISD $=3.46$ and 8.66 $\mathrm{km}$ respectively, by each eNodeB for the DVB flows' repair via LTE. We present these results for the universal population density i.e. 100 inhabitants per sq.km. The weakest signal level of the DVB SFN is not located in the edges of the central cell, but actually at some point before the edge. It can be observed that in the troubled zones the deployment of a multicast repair operation keeps eNodeBs 8 to 15 times less and smoothly loaded compared to unicast repair for the respective ISD values analyzed.

\section{CONCLUSION}

In this paper we have extended the concept of service level end-to-end convergence between the conventional Terrestrial Video Broadcast and the cellular networks. Our work primarily thrives on the parity signal i.e. SRQC overhead packets generation in the LTE network and its multicast based distribution in the access network. Based on the conventional SRQC coding, we have considered the possibility of generating parity strings for the recovery of a large number of erased BB-frames from the DVB transmission. The LTE eMBMS multicast bearers have been deployed using concentric MBSFNs while assuming very pessimistic conditions. The multicast-based repair operation over the DVB broadcast flows was found to be resourceeconomical compared to the unicast retransmission. Our results demonstrate that implementing a multicast bearer based repair for DVB flows via LTE network with a low impact overhead, enhances the scope of a cooperative framework for better quality of multimedia multicast services to handheld receivers.

\section{REFERENCES}

[1] I. Elizmendi, G. Prieto, G. Berjon-Eriz, I. Pena, M. M. Velez, and Pablo Angueira. Next generation of broadcast multimedia services to mobile receivers in urban environments. Elsevier Signal Processing: Image Communication, special issue on pervasive mobilemultimedia, 27:925-933, Sep. 2012.

[2] I. Eizmendi, M. Velez, D. Gomez-Barquero, J. Morgade, V. Baena-Lecuyer, M. Slimani, and J. Zoellner. Dvbt2: The second generation of terrestrial digital video broadcasting system. Broadcasting, IEEE Transactions on, 60(2):258-271, June 2014.

[3] A160. Digital Video Broadcasting (DVB); Next Generation broadcasting system to Handheld, physical layer specification (DVB-NGH), November 2012.

[4] D. Gomez-Barquero, C. Douillard, P. Moss, and V. Mignone. Dvb-ngh: The next generation of digital broadcast services to handheld devices. Broadcasting, IEEE Transactions on, 60(2):246-257, June 2014.

[5] M.M. Anis, X. Lagrange, and R. Pyndiah. Cellular-based real-time flow repair for broadcast flows. Broadcasting, IEEE Transactions on, 61(3):457-469, Sept 2015.

[6] Amin Zribi, Ramesh Pyndiah, Samir Saoudi, and Xavier Lagrange. Erasure coding for reliable adaptive retransmission in wireless broadcast/multicast systems. Springer: Telecommunication Systems, 65(4):591-604, August 2017.

[7] 3GPP Technical Specification. TS 23.246 Group Services and System Aspects; Multimedia Broadcast/Multicast Service (MBMS) Architecture and functional description (Release 13), v13.3.0 edition, 2015.

[8] J. Skold E. Dahlman, S. Parkvall. 4G: LTE/LTEAdvanced for Mobile Broadband. Elsevier Inc., first edition, 2011.

[9] 3GPP. Technical Specification Group Services and System Aspects; Vocabulary for 3GPP Specifications (Release 10). TR 21.905 V10.0.0, 2010.

[10] A133. "Implementation guidelines for a second generation digital terrestrial television broadcasting system (DVB-T2)", 2010.

[11] Amin Shokrollahi. Raptor codes. Information Theory, IEEE Transactions on, 52(6):2551, June 2006.

[12] Xiaoli Wang, Yongsheng Zhang, and Song Xiao. How network coding helps embms. In IET International Conference on Communication Technology and Application (ICCTA 2011), pages 511-515, Oct 2011.

[13] Micheal Luby, Amin Shokrollahi, Mark Watson, and Thomas Stockhammer. draft-ietf-rmt-bb-fec-raptorq-02. RaptorQ Forward Error Correction Schemd for Object Delivery, IETF Anaheim(77), March 2010.

[14] Tracey Ho. Summary of raptor codes. Pre-print of Raptor Codes by Amin Shokrollahi, October 2003.

[15] T. Pail, M. Luby, R. Walsh, V. Roca, and R. Lehtonen. Flute - file delivery over unidirectional transport. IETF RFC 6726, November 2012.

[16] M. M. Anis, X. Lagrange, and R. Pyndiah. A simple model for the dvb and le cooperation. In IEEE 8th Broadband Multimedia Systems and Broadcasting (BMSB) Symposium, 2013., June 2013.

[17] ETSI. "EN 302 755: Frame structure channel coding and modulation for a second generation digital terrestrial television broadcasting system", v1.3.1 edition, 2012.

[18] EBU UER Technical Report. EBU - TECH 3348: Frequency and Network Planning aspects of DVB-T2, 2011.

[19] A. Abdel-Razzac, S. Elayoubi, T. Chahed, and B. El Hassan. Impact of lte and dvb-ngh cooperation on qos of mobile tv users. In International Conference on Communications 2013 (ICC 2013), pages 1-5, 2013.

[20] P. Mogensen, Wei Na, I.Z. Kovacs, F. Frederiksen, A. Pokhariyal, K.I. Pedersen, T. Kolding, K. Hugl, and M. Kuusela. LTE Capacity Compared to the Shannon Bound. In Vehicular Technology Conference, 2007. VTC2007-Spring. IEEE 65th, pages $1234-1238$, april 2007. 